\title{
Differential gene expression signatures for cell wall integrity found in chitin synthase II (chs2 $\Delta$ ) and myosin II (myol $\Delta$ ) deficient cytokinesis mutants of Saccharomyces cerevisiae José F Rodríguez-Quiñones and José R Rodríguez-Medina*
}

\author{
Address: Department of Biochemistry, School of Medicine, University of Puerto Rico, Medical Sciences Campus, San Juan, Puerto Rico \\ Email: José F Rodríguez-Quiñones - francquimico@gmail.com; José R Rodríguez-Medina* - jose.rodriguez123@upr.edu \\ * Corresponding author
}

Published: 9 May 2009

BMC Research Notes 2009, 2:87 doi:10.1 186/1756-0500-2-87

This article is available from: http://www.biomedcentral.com//756-0500/2/87

(c) 2009 Rodríguez-Medina et al; licensee BioMed Central Ltd.

This is an Open Access article distributed under the terms of the Creative Commons Attribution License (http://creativecommons.org/licenses/by/2.0), which permits unrestricted use, distribution, and reproduction in any medium, provided the original work is properly cited.

\begin{abstract}
Background: Myosin II-dependent contraction of the cytokinetic ring and primary septum formation by chitin synthase II are interdependent processes during cytokinesis in Saccharomyces cerevisiae. Hence, null mutants of myosin II (myol $\Delta$ ) and chitin synthase II (chs2 $\Delta$ ) share multiple morphological and molecular phenotypes. To understand the nature of their interdependent functions, we will seek to identify genes undergoing transcriptional regulation in chs $2 \Delta$ strains and to establish a transcription signature profile for comparison with myol $\Delta$ strains.
\end{abstract}

Results: A total of 467 genes were commonly regulated between myo $\Delta$ and chs $2 \Delta$ mutant strains $(\mathrm{p} \leq 0.0 \mathrm{I})$. Common regulated biological process categories identified by Gene Set Enrichment Analysis (GSEA) in both gene expression profiles were: protein biosynthesis, RNA processing, and stress response. Expression of $17 / 20$ genes in the main transcriptional fingerprint for cell wall stress was confirmed in the chs $2 \Delta$ strain versus $5 / 20$ for the myol $\Delta$ strain. One of these genes, SLT2/ $M P K I$, was up-regulated in both strains and both strains accumulated the hyperphosphorylated form of SIt2p thereby confirming that the $\mathrm{PKCl}$ cell wall integrity pathway (CWIP) was activated by both mutations. The SLT2IMPKI gene, essential for myol $\Delta$ strains, was not required in the chs $2 \Delta$ strain.

Conclusion: Comparison of the chs $2 \Delta$ and myo $/ \Delta$ gene expression profiles revealed similarities in the biological process categories that respond to the chs $2 \Delta$ and myol $\Delta$ gene mutations. This supports the view that these mutations affect a common function in cytokinesis. Despite their similarities, these mutants exhibited significant differences in expression of the main transcriptional fingerprint for cell wall stress and their requirement of the CWIP for survival.

\section{Background}

In the budding yeast Saccharomyces cerevisiae, myosin type II (Myo1p) and chitin synthase II (Chs2p) are essential proteins for the formation of the normal cytokinetic apparatus. These proteins participate in assembly of the cytokinetic ring and synthesis of the primary septum, respectively, during cytokinesis [1-3]. Previous studies revealed that contraction of the cytokinetic ring and closure of the primary septum by Chs2p are interdependent processes that occur at adjacent sites on the plasma membrane and at similar times of the cell cycle [3]. Previous studies also showed that Chs $2 \mathrm{p}$ at the bud neck is required 
to maintain the stability of the actomyosin ring and for normal completion of the cytokinetic process $[2,4]$. Thus, MYO1 and CHS2 deficient cells, (myo1 $\Delta$ and chs $2 \Delta$ respectively), have been described to be cytokinesis mutant strains [5] that share multiple phenotypes associated with their respective cytokinesis defect [3]. Both mutants require expression of chitin synthase III (Chs3p), [3,6,7] an enzyme that synthesizes $\sim 90 \%$ of the cell wall chitin, which implies the existence of cell wall stress conditions. Cell wall stress can be overcome by activation of the PKC1-dependent cell wall integrity pathway [8,9]. Readouts of the pathway in yeast cells include up-regulation of cell wall biosynthetic enzymes, heat shock proteins, and increased production of other cell wall components [8].

In this study we will establish a signature transcription profile for chs $2 \Delta$ strains and compare it with the signature profile in myo1s strains previously published [10]. To accomplish this goal, we have performed a comparative oligonucleotide microarray analysis of mRNAs extracted from a chs $2 \Delta$ strain and wild-type controls. When compared to the profiles previously reported in myo1 $\Delta$ strains, we observed that both mutants alter the expression of similar groups of genes associated with specific biological process categories. However, these mutants exhibit significant differences in expression of the main transcriptional fingerprint for cell wall stress and differ in their requirement of the cell wall integrity pathway for survival.

\section{Methods}

\section{Strains and culture conditions}

All the experiments were performed using Saccharomyces cerevisiae wild type, chs $2 \Delta$, and myo1 $\Delta$ strains (Table 1 ). Cultures were grown overnight at $26^{\circ} \mathrm{C}$ between $0.5-0.8$ $\mathrm{OD}_{600}$ in Complete Synthetic Medium (CSM)(2\% glucose, $1 \times$ Nitrogen base), and CSM-HIS- with continuous shaking at $200 \mathrm{rpm}$.

\section{SLT2 gene disruption}

A SLT2 gene disruption was created in the myo1 1 pRS316MYO1 strain (Table 1) by replacing the SLT2 gene with a KanMX4 module by homologous recombination. The myo1s slt2 $\Delta$ pRS316-MYO1 strain was grown in CSM 5FOA to uncover the myo1 sslt2 $\Delta$ mutant [10]. Mutant chs $2 \Delta$ strains were transformed with URA3 cassette, to perform the SLT2 gene disruption. All colonies were confirmed by PCR.

\section{RNA extraction procedure}

Total RNA was extracted from cells derived from five biological replicate cultures of wild type and chs $2 \Delta$ strains as described previously [10]. RNA concentrations, purity and integrity were determined using a Nanodrop spectrophotometer (Nanodrop Technologies, Wilmington, DE) and an Agilent Bioanalyzer (Agilent Technologies, Palo Alto, CA) respectively.

\section{Oligonucleotide microarray experiments}

Oligonucleotide microarray experiments and data analysis were performed as described previously [10]. Briefly, $1.0 \mu \mathrm{g}$ of total RNA from each sample was amplified using the Low RNA Input Fluorescent Linear Amplification kit (Agilent Technologies, Palo Alto, CA), and then it was labeled with $10 \mathrm{mM}$ Cy3 or Cy5. Labeled cRNA's were hybridized, and then microarray slides were washed and scanned with a VersArray Chip Reader (BioRad, Hercules, $\mathrm{CA})$. The microarrays raw data was generated with Imagene 3.0 and then analyzed using Limma software [11] as previously described [10]. The fold change in gene expression was calculated by $2(\mathrm{M})$, where $M$ is the $\log _{2}$-fold change after background correction and normalization. An Empirical Bayes Statistics [12]for differential expression analysis and FDR test [13] were performed. The pvalue $\leq 0.01$ cutoff was established for differential expression. Gene Set Enrichment Analysis (GSEA) [14] was performed using the Limma package of Bioconductor as described previously [10]. A corrected p-value was obtained from the analysis using the Bonferroni correc-

Table I: Strains used in this study.

\begin{tabular}{|c|c|c|}
\hline Strain & Genotype & Source \\
\hline $\begin{array}{l}\text { MGD353-46D } \\
\text { (wild type) }\end{array}$ & MAT $\alpha$ trp I-289 ura3-52 leu2-3, II 2 his3deltal $\mathrm{ADE}^{+}$ARG cyh ${ }^{R}$ & B. Rymond \\
\hline $\begin{array}{l}\text { YJR6 } \\
(m y o l \Delta)\end{array}$ & $\begin{array}{l}\text { MAT } \alpha \text { trp I-289 ura3-52 leu2-3, II } 2 \text { his3deltal ADE } \text { ARG cyh }^{R} \text { myo I delta::HIS5+ parental MGD353- } \\
\text { 46D }\end{array}$ & F. Rivera \\
\hline $\begin{array}{l}\text { YFR23 } \\
(\text { chs } 2 \Delta)\end{array}$ & MAT $\alpha$ trp I-289 ura3-52 leu2-3, II 2 his3deltal ADE ${ }^{+}$ARG cyh ${ }^{R}$ chs2delta::KANR parental MGD353-46D & F. Rivera \\
\hline $\begin{array}{l}\text { YJF2 } \\
(\text { chs } 2 \Delta s / t 2 \Delta)\end{array}$ & $\begin{array}{l}\text { MAT } \alpha \text { trp I-289 ura3-52 leu2-3, I I } 2 \text { his3deltal ADE }{ }^{+} \text {ARG cyhRchs2delta::KANRslt2delta::URA3+ } \\
\text { parental MGD353-46D }\end{array}$ & This study \\
\hline $\begin{array}{l}\text { YJF9 } \\
(\text { myol } \Delta s I t 2 \Delta \text { pRS3I6-MYOI) }\end{array}$ & $\begin{array}{l}\text { MAT } \alpha \operatorname{trp} 1-289 \text { ura3-52 leu2-3, II } 2 \text { his3deltal } \mathrm{ADE}^{+} \text {ARG cyh }{ }^{R} \text { myol delta::HIS5+ parental MGD353- } \\
\text { 46D, pRS3I6-MYOI, slt2delta::KAN }\end{array}$ & This study \\
\hline $\begin{array}{l}\text { YJF8 } \\
(s / t 2 \Delta)\end{array}$ & 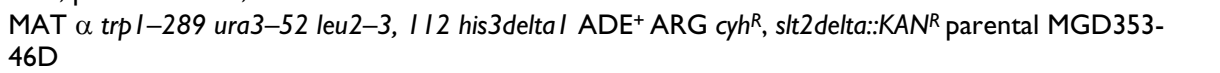 & This study \\
\hline
\end{tabular}


tion $\mathrm{p}$-value $\leq 0.0004$. Microarray raw and processed data are available at the Gene Expression Omnibus (GEO) site

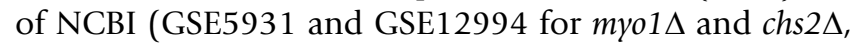
respectively) [15].

\section{Real time RT-PCR experiments}

Real time RT-PCR assays were performed with $30 \mathrm{ng}$ of total RNA using the Quantitec SYBR Green RT-PCR kit (Qiagen, Valencia, CA) as previously described [10]. The sequences of the forward and reverse primers for the selected mRNAs are listed in Table 2 . The fold change was determined by the $2^{\Delta \Delta \mathrm{Ct}}$ method [16] as described previously [10].

Western blot analysis of hyperphosphorylated Slt2p levels Yeast strains were grown in selective medium between $0.5-0.8 \mathrm{OD}_{600}$ at $26^{\circ} \mathrm{C}$. Cells treatment, protein extraction and quantification methods were performed as described $[6,9,10,17]$. Total protein extracts $(75 \mu \mathrm{g})$ were separated in a 10\% SDS-PAGE gel and transferred to a nitrocellulose membrane at $70 \mathrm{~V}$ for $2 \mathrm{~h}$ at $4{ }^{\circ} \mathrm{C}$. The membrane was incubated with anti-phospho-p42/44 MAP kinase monoclonal antibody (1:1000) (Cell Signaling Technologies, Danvers, MA). The membrane was stripped and reprobed with a rabbit polyclonal antibody against Slt2p (1:1000) and mouse monoclonal antibody against Pgk1p (1:500) (Molecular probes, Invitrogen, Danvers, $\mathrm{MA})$.

\section{Results and Discussion Comparison between transcriptional profiles of $\mathrm{chs} 2 \Delta$ and myol $\Delta$ strains}

The rationale for these experiments was to identify differentially expressed genes and common biological process categories that are relevant to the myosin-dependent versus myosin-independent cytokinesis mechanisms operating in chs $2 \Delta$ and myo1s strains respectively. Microarray hybridization experiments were conducted as described previously [10] with labeled total RNA obtained from five independent biological replicate cultures of the chs $2 \Delta$ and wild type control strains (Table 1). A total of 467 genes were differentially expressed in common between chs $2 \Delta$ and myo1s strains $(\mathrm{p} \leq 0.01)$ and classified according to their biological processes [see Additional file 1]. The results of the oligonucleotide microarrays for the chs $2 \Delta$ strain were validated by real time RT-PCR indicating a fold change of $1.11,2.92,7.21,1.41$, and 1.11 for SLT2, ECM4, SPI1, YHR097C, and ROM1 respectively that were consistent with the microarray results (Table 3 ).

A comparison of results from GSEA of $c h s 2 \Delta$ with those previously published for myo1s strains [10] revealed similar yet distinct transcription signature profiles. Five categories were identified with a corrected $\mathrm{p}$-value below the cutoff ( $\mathrm{p} \leq 0.0004$ ) for chs $2 \Delta$ strains (Figure 1A, top and bottom panels). These categories were: protein biosynthesis, stress response, RNA processing, autophagy and genes encoding unknown biological processes. Histograms of density versus t-value were generated for each category to determine if regulation of a specific category occurred by comparing the distribution of genes in each biological process category relative to the normal distribution of all the categories represented on the array (Figure 1A, top panel). Plots of t-values versus A-values were created to identify the individual genes in each category and observe their distribution across the array (Figure 1A, bottom panel). Of the five biological process categories selected, the protein biosynthesis and RNA processing categories presented the most dramatic changes in their normal distribution (Figure 1A, top panel). The histogram reflecting the tendency of the protein biosynthesis category showed a shift from the normal distribution towards negative $\mathrm{t}-$ values. This was also observed in the corresponding $\mathrm{t}$ value vs. A-value plot where we observe a greater quantity of genes biased towards negative t-values (Figure 1A, bottom panel). In the case of the RNA processing category, these genes were also shifted towards negative t-values (Figure 1A, top panel). These results are consistent with those obtained previously in myo1s mutant strains where the protein biosynthesis and RNA processing categories

Table 2: Primers used in this study for real time RT-PCR and genetic deletions

\begin{tabular}{cll}
\hline Target & Forward primer & Reverse primer \\
\hline ACTI & 5'-GCCATTTTGAGAATCGATTTG-3' & 5'-TTAGAAACACTTGTGGTGAAC-3' \\
ECM4 & 5'-GTGGTACAAACGGAGCTTTCA-3' & 5'-GTGCCCAATGGACTACGCTACA-3' \\
SPII & 5'-CCAGAACCAACGACTTTCGTA-3' & 5'-ACTGCACCAGCCAAACCTA-3' \\
ROMI & 5'-AGCTATCTACGCCTCCAACT-3' & 5'-ATGATGACGTTGGTGTTGA-3' \\
SLT2 & 5'-AGCAACAGCAGCCTTCAGA-3' & 5'-GAACGCGAGGAAGTATCCAA-3' \\
YHR097C & 5'-CCATCGTCGTACATCACAC-3' & 5'-GTACAGGCGCCACTTTATTA-3' \\
SLT2pRS & 5'-AATGGAAAGTTTCAGTGTTAAAAATAG & 5'-AATAATGAATATTGTCTATAGATGACTAA \\
& AAACTGAAAAAGGAGATCTAGCCCGTTTCGGTGATGAC-3' & TCATAAATGAACGAAAAGAAATTCCTGATGCGGTATTTTC \\
ATG9PRS & 5'-TTACACTAATTAAGATGCTTAGATTCC & TCC-3' \\
& CATTCAAAAGGTACTATTGACGTCGTTTCGGTGATGAC-3' & 5'-GGCGGAAAGATAATTAACATAACCAATAATTATTTTCTTTTCCT \\
& & GATGCGGTATTTTCTCCT-3'
\end{tabular}


Table 3: Confirmation of microarray data by real time RT-PCR assay on a selected set of genes for chs $2 \Delta$ and $m y \circ I \Delta[10]$ (p $\leq 0.0 I$ )

\begin{tabular}{ccccc}
\hline Gene name & $\begin{array}{c}\text { Fold Change in Microarray } \\
\text { myo I } \Delta[\mathrm{I0}]\end{array}$ & Fold Change in Microarray chs2 & $\begin{array}{c}\text { Fold Change } \\
\text { (real time RT-PCR) } m y 0 I \Delta[10]\end{array}$ & $\begin{array}{c}\text { Fold Change } \\
\text { (real time RT-PCR) chs2 } \Delta\end{array}$ \\
\hline SLT2 & 2.1 & 1.30 & 1.8 & 1.11 \\
ECM4 & 3.2 & 2.13 & 3.5 & 2.92 \\
SPII & 8.6 & 1.94 & 13.0 & 7.21 \\
YHRO97C & 4.1 & 2.01 & 3.8 & 1.41 \\
ROMI & 3.2 & 1.30 & 3.5 & 1.11 \\
\end{tabular}

were biased towards negative $\mathrm{t}$-values (Figure 1B top and bottom panels). Because levels of ribosome biogenesis and growth rate are correlated in budding yeast $[18,19]$, down-regulation of protein biosynthesis genes is an indication that growth rate is also reduced, a phenotype that has been reported previously in both mutant strains $[2,10]$. Although the "unknown" biological process category had the greatest representation of differentially expressed genes in the array (670 genes), there was no shift observed in the density vs. t-values histogram or the $\mathrm{t}$-values vs. A-values plot for chs $2 \Delta$ (Figure 1A) suggesting that a similar numbers of genes were up and down regulated. The myo1 $\Delta$ and $c h s 2 \Delta$ mutant profiles differed in the autophagy and carbohydrate biological process categories.

\section{Analysis of the $\mathrm{PKCl}$-dependent cell wall integrity pathway requirement}

A previous study of myo1 1 strains revealed that the PKC1dependent pathway was activated by up-regulation of the SLT2 gene and an increase in hyperphosphorylated Slt2p (p-Slt2p) at steady state compared to their wild type $[6,10,17]$. In the chs $2 \Delta$ gene expression profile, the SLT2 gene was also up-regulated (1.3-fold), and p-Slt2p levels were increased, thereby revealing $P K C 1$ activation in this mutant (Figure 2A). However, the slt $2 \Delta$ knockout mutation was viable in a chs $2 \Delta$ strain although it was previously shown to be lethal in a myo1 $\Delta$ strain (Figure 2B). The slt2 $\Delta$ knockout mutation was confirmed in the chs $2 \Delta$ strain by PCR (data not shown) and reconfirmed at the protein level by western blot (Figure 2A). These observations suggest that activation of the PKC1-dependent cell wall integrity pathway for survival is a myo1 1 -specific phenotype and may indicate that cell integrity defects are more severe in this strain.

A previous study described a transcriptional fingerprint consisting of 20 core genes that are a characteristic for cell wall stress caused by perturbation of cell wall integrity $[20,21]$. That study included the fks $1 \Delta$ strain, which is considered a typical cell wall-deficient mutant and is characterized by the transcriptional up-regulation of the SLT2 gene and increase in the hyperphosphorylated form of Slt2p $[20,21]$. We performed confirmatory experiments in an $f k s 1 \Delta$ strain and observed transcriptional activation of
17 of the described 20 core genes, thereby corroborating their results (data not shown). Despite the dispensability of Slt $2 p$, the chs $2 \Delta$ strain expressed 17 of the 20 core genes mentioned, in contrast to only 5 core genes activated in the myo1 $\Delta$ strain (Table 4 ). Such a difference in transcription of these genes suggests that these two mutations affect cell wall integrity at different levels.

\section{Conclusion}

In this study the global mRNA expression profile of a chs $2 \Delta$ mutant strain was determined and analyzed by GSEA for further comparison with the previously reported myo1 $\Delta$ strain profile [10]. The GSEA identified four biological categories affected by each mutant condition, where protein biosynthesis and RNA processing categories were down- regulated while stress response and autophagy were up-regulated. This analysis revealed that genes involved in the autophagy process were significantly up-regulated exclusively in the chs $2 \Delta$ strain. We did not detect any growth impairment in an autophagy-deficient strain $\operatorname{chs} 2 \Delta \operatorname{atg} 9 \Delta$ (data not shown) suggesting that although this represented a differentially regulated biological process in the chs $2 \Delta$ strain, autophagy was not essential for growth of this mutant under our culture conditions.

We have demonstrated that the PKC1-dependent cell integrity pathway was activated in both myo1 $1 \Delta$ and chs $2 \Delta$ strains. Slt2p/Mpk1p, a gene product of the signal transduction biological process category, was not essential for cell viability in the chs $2 \Delta$ mutant. Nonetheless, the chs $2 \Delta$ strain exhibited higher resistance to cell lysis by exogenously added $\beta-1,3$ glucanase than a wild-type strain suggesting that it most likely has modified its cell wall composition (data not shown). A positive contribution of the cell wall integrity pathway to these putative modifications may be inferred from the transcriptional fingerprint for this mutant.

In summary, a comparison of the chs $2 \Delta$ and $m y 01 \Delta$ gene expression profiles revealed similarities in the biological process categories that respond to the chs $2 \Delta$ and myo1s gene mutations. This supports the view that these mutations may affect common functions in cytokinesis. Despite their similarities, these mutants exhibited signifi- 
A)
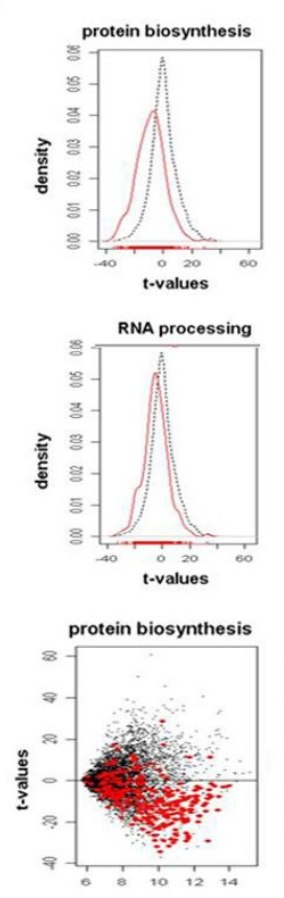

A-values

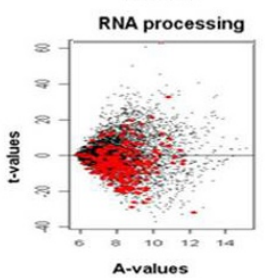

chs2 $\Delta$
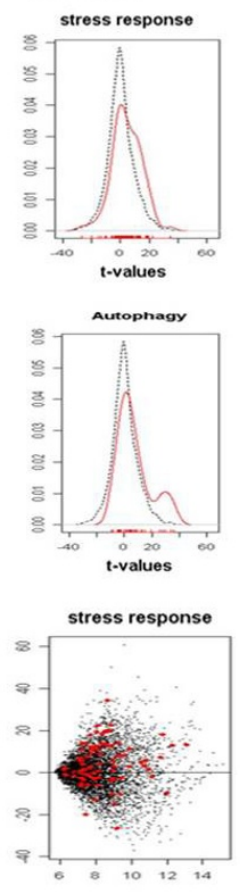

A-values

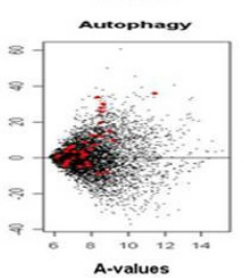

B)
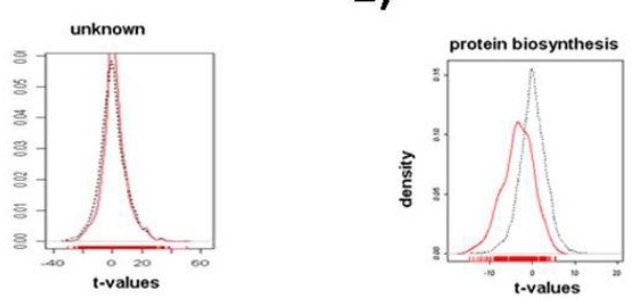

myo1 $\Delta^{*}$
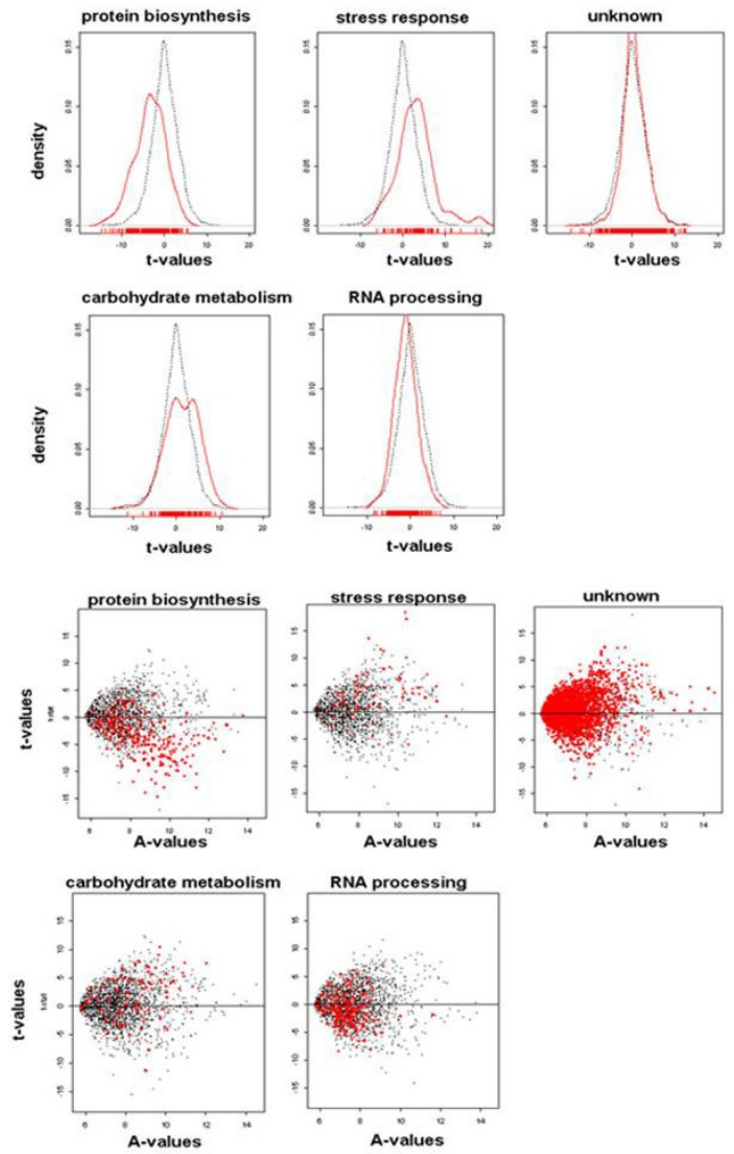

*BMC Genomics 2008, 9:34

\section{Figure I}

Histograms derived from Gene Set Enrichment Analysis (GSEA) of chs $2 \Delta$ and myol $\Delta$ mutant strains. The cutoff for significantly regulated biological process categories was based on a $p$-value $\leq 0.0004$ as described in the Methods section. Red lines or dots represent the distribution of genes of the specific category in the array. Black lines or dots represent the distribution of genes for all categories in the array. (A, top panel) Density versus t-value plots generated for significant biological process categories from the chs $2 \Delta$ strain. (A, bottom panel) t-value versus A-value plots for genes of the same biological process categories shown in (A, top panel). (B, top panel) Density versus t-value plots previously generated for significant biological process categories from the myol $\Delta$ strain $[10]$. (B, bottom panel) t-value versus A-value plots for genes of the same biological process categories shown in (B, top panel). The results shown in Figure IB were previously published in BMC Genomics 2008, 9:34 [10].

cant differences in expression of the main transcriptional fingerprint for cell wall stress and in their requirement of these genes for survival. These differences provide insight to how S. cerevisiae circumvents cell death and may help in the development of novel antifungal treatment.

\section{Competing interests}

The authors declare that they have no competing interests.

\section{Authors' contributions}

JFRQ participated in the microarray experiments and data analysis, real-time RT-PCR experiments, western blot experiments, and writing of the manuscript. JRRM participated as principal investigator in the design of experiments, analysis and interpretation of the data, and writing of the manuscript. All authors read and approved the final manuscript. 
A)

B)

\section{CSM media}

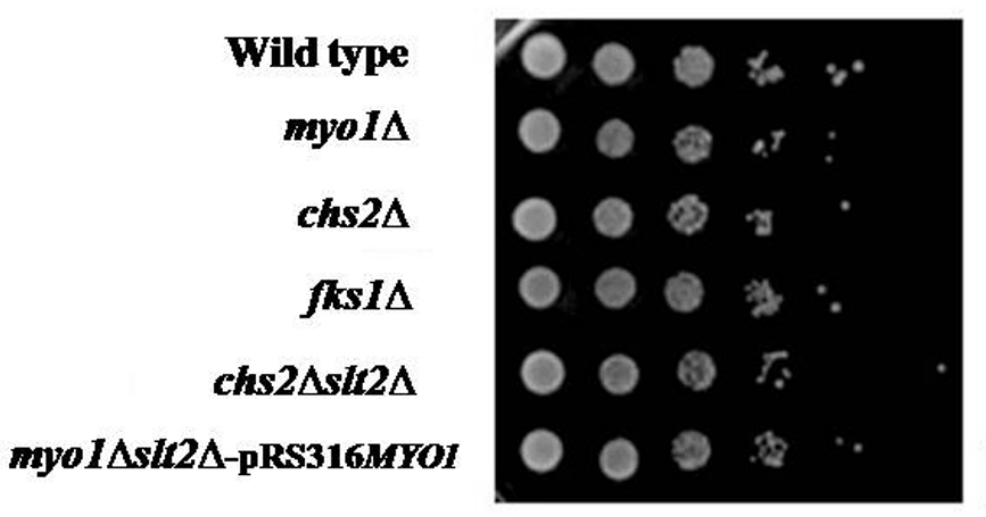

\section{CSM 5-FOA media}

Figure 2

Analysis of the $P K C I$-dependent cell wall integrity pathway in chs $2 \Delta$ strains. A) Western blot assay for activation of the $P K C l$-dependent cell wall integrity pathway in chs $2 \Delta$ strains. Equal amounts of protein $(75 \mu \mathrm{g})$ from wild type, myol $\Delta$, chs $2 \Delta$, sit2, and chs $2 \Delta$ sit2 $\Delta$ strains were analyzed by western blot to determine steady state levels of hyperphosphorylated SIt2p ( $\mathrm{p}$-Slt2p), total levels of Slt2p, and PgkIp (a loading control) as described in the Methods section. B) Viability assays for wild type, myol $\Delta$, chs $2 \Delta$, slt2 $\Delta$, chs $2 \Delta s / t 2 \Delta$, and myo I $\Delta$ slt $2 \Delta \mathrm{pRS} 316-M Y O I$ strains. Serial dilutions ranging from I $\times 10^{7}-\mathrm{I} \times 10^{2}$ cells $/ \mathrm{ml}$ were plated on CSM media to observe growth after three days of culture at $29^{\circ} \mathrm{C}$. The myo I $\Delta$ slt $2 \Delta$ mutant was maintained by co-expressing a plasmid-borne copy of the MYOI gene (pRS3I6-MYOI) as described previously [I0]. Synthetic lethality was uncovered in this strain by negative selection for URA3 (contained on the plasmid) with 5-FOA. 
Table 4: Twenty genes representing the main transcriptional fingerprint for cell wall stress [20].

\begin{tabular}{lll}
\hline Gene name & chs2 & myol $[$ [I0] \\
\hline YLRI94C & Up-regulated & \\
YPL088W & & \\
YIL023C & Up-regulated & \\
YLR4I4C & Up-regulated & \\
YHR097C & Up-regulated & Up-regulated \\
YAL053W & Up-regulated & \\
CWPI & Up-regulated & \\
SEDI & Up-regulated & Up-regulated \\
PIR3 & Up-regulated & \\
CRHI & Up-regulated & \\
KTR2 & Up-regulated & \\
GFAI & Up-regulated & \\
PSTI & Up-regulated & \\
SLT2 & Up-regulated & Up-regulated \\
MLPI & Up-regulated & \\
YPS4 & & \\
FBP26 & Up-regulated & Up-regulated \\
HSPI2 & Up-regulated & Up-regulated \\
PRM5 & Up-regulated & \\
SRL3 & & \\
\hline
\end{tabular}

Blank boxes signify no change in expression.

\section{Additional material}

\section{Additional File 1}

Differentially expressed genes found in common between myo1 $\triangle$ and chs2 $\triangle$ strains $(p \leq 0.01)$.

Click here for file

[http://www.biomedcentral.com/content/supplementary/17560500-2-87-S1.pdf]

\section{Acknowledgements}

The authors thank Dr. Rafael Irizarry for continuous advice in microarray data analysis during the course of JFRQ's graduate training. We also thank Sahily González-Crespo and Lilliam Villanueva-Alicea for outstanding technical support. This work was supported by a USPHS grant to JRRM from NIGMS/NIAID (ISCI AI08I 658-0I) with partial support from NCRR-RCMI (GI2RR0305I). JFRQ received support from MBRS-RISE (R25GM6I838).

\section{References}

I. Bi E, Maddox P, Lew DJ, Salmon ED, McMillan JN, Yeh E, Pringle JR: Involvement of an actomyosin contractile ring in Saccharomyces cerevisiae cytokinesis. / Cell Biol I998, I 42(5): | 30|-1312.

2. Silverman SJ, Sburlati A, Slater ML, Cabib E: Chitin synthase $\mathbf{2}$ is essential for septum formation and cell division in Saccharomyces cerevisiae. Proc Natl Acad Sci USA 1988, 85(I 3):4735-4739.

3. Schmidt M, Bowers B, Varma A, Roh DH, Cabib E: In budding yeast, contraction of the actomyosin ring and formation of the primary septum at cytokinesis depend on each other. J Cell Sci 2002, I I 5(Pt 2):293-302.

4. VerPlank L, Li R: Cell cycle-regulated trafficking of Chs2 controls actomyosin ring stability during cytokinesis. Mol Biol Cell 2005, I 6(5):2529-2543.

5. Rodriguez JR, Paterson B: Yeast Myosin Heavy Chain Mutant: Maintenance of the Cell Type Specific of Chitin and Cell Wall Components Requires an Intact Myosin Heavy Chain. Cell Motil Cytoskel 1990, I7:30 I-308.
6. Rivera-Molina FE: Biochemical and Genetic Analysis of Myosin II Function in the Trafficking of the Chitin Synthase III Catalytic Subunit. In Ph.D. Thesis University of Puerto Rico Medical Sciences Campus; 2005.

7. Rivera-Molina F, Gonzalez-Crespo S, Maldonado-de la Cruz Y, OrtizBetancourt JM, Rodriguez-Medina JR: 2,3-Butanedione monoxime increases sensitivity to Nikkomycin $Z$ in the budding yeast Saccharomyces cerevisiae. World J Microbio Biotechnol 2006, 22:255-260.

8. Levin DE: Cell wall integrity signaling in Saccharomyces cerevisiae. Microbiol Mol Biol Rev 2005, 69(2):262-291.

9. de Nobel H, Ruiz C, Martin H, Morris W, Brul S, Molina M, Klis FM: Cell wall perturbation in yeast results in dual phosphorylation of the SIt2/MpkI MAP kinase and in an SIt2-mediated increase in FKS2-lacZ expression, glucanase resistance and thermotolerance. Microbiology 2000, 146(Pt 9):2|2|-2|32.

10. Rodriguez-Quinones JF, Irizarry RA, Diaz-Blanco NL, Rivera-Molina FE, Gomez-Garzon D, Rodriguez-Medina JR: Global mRNA expression analysis in myosin II deficient strains of Saccharomyces cerevisiae reveals an impairment of cell integrity functions. BMC Genomics 2008, 9:34

II. Gentleman RC, Carey VJ, Bates DM, Bolstad B, Dettling M, Dudoit S, Ellis B, Gautier L, Ge Y, Gentry J, et al.: Bioconductor: open software development for computational biology and bioinformatics. Genome Biol 2004, 5(I 0):R80.

12. Smyth G: Linear models and empirical Bayes methods for assessing differential expression in microarray experiments. Stat Appl Genet Mol Biol 2004, 3:Article3.

13. Benjamini Y, Hochberg Y: Controlling the False Discovery Rate: A practical and powerful approach to multiple testing. JR Statist Soc B 1995, 57:289-300.

14. Mootha VK, Lindgren CM, Eriksson KF, Subramanian A, Sihag S, Lehar J, Puigserver P, Carlsson E, Ridderstrale M, Laurila E, et al.: PGCIalpha-responsive genes involved in oxidative phosphorylation are coordinately downregulated in human diabetes. Nat Genet 2003, 34(3):267-273.

15. Barrett T, Troup DB, Wilhite SE, Ledoux P, Rudnev D, Evangelista C, Kim IF, Soboleva A, Tomashevsky M, Edgar R: NCBI GEO: mining tens of millions of expression profiles-database and tools update. Nucleic Acids Res 2007:D760-765.

16. Livak KJ, Schmittgen TD: Analysis of relative gene expression data using real-time quantitative PCR and the 2(-Delta Delta C(T)) Method. Methods 200I, 25(4):402-408.

17. Diaz-Blanco NL, Rodriguez-Medina JR: Dosage rescue by UBC4 restores cell wall integrity in Saccharomyces cerevisiae lacking the myosin type II gene MYOI. Yeast 2007, 24(4):343-355.

18. Hughes TR, Marton MJ, Jones AR, Roberts CJ, Stoughton R, Armour $\mathrm{CD}$, Bennett HA, Coffey E, Dai H, He YD, et al.: Functional discovery via a compendium of expression profiles. Cell 2000, 102(I): 109-126.

19. Levy S, Ihmels J, Carmi M, Weinberger A, Friedlander G, Barkai N: Strategy of transcription regulation in the budding yeast. PLoS ONE 2007, 2(2):e250.

20. Garcia R, Bermejo C, Grau C, Perez R, Rodriguez-Pena JM, Francois J, Nombela $C$, Arroyo J: The global transcriptional response to transient cell wall damage in Saccharomyces cerevisiae and its regulation by the cell integrity signaling pathway. J Biol Chem 2004, 279(15): 15183-15195.

21. Lagorce A, Hauser NC, Labourdette D, Rodriguez C, Martin-Yken H, Arroyo J, Hoheisel JD, Francois J: Genome-wide analysis of the response to cell wall mutations in the yeast Saccharomyces cerevisiae. J Biol Chem 2003, 278(22):20345-20357. 